\title{
Analytical chemistry for infectious disease detection and prevention
}

\author{
XiuJun $\mathrm{Li}^{1,2}$ (D) Chaoyong Yang ${ }^{3}$
}

Received: 27 May 2021 / Accepted: 27 May 2021 / Published online: 5 June 2021

(C) Springer-Verlag GmbH Germany, part of Springer Nature 2021

Infectious diseases refer to disorders caused by different pathogens, including bacteria, viruses, fungi, and parasites. They can rapidly transmit from person to person, and quickly spread from one location throughout the whole world. Therefore, their impacts are far-reaching; they often cause tremendous global health concerns and economic losses. Some examples of those infectious diseases include COVID-19, SARS, Ebola, HIV, tuberculosis (TB), malaria, and meningitis. In particular, the ongoing pandemic caused by COVID-19 has reminded us of the severity and impacts of infectious disease on our society. As of May 14, 2021, the statistics from John Hopkin University shows that there are about 161,482,418 COVID19 cases, and 3,351,517 associated deaths worldwide. This has caused intensive global attention. Using "COVID-19" as the keyword to search from Web of Science on the same day, we found more than 111,000 items were published in 1.5 years, and 4294 of them $(6.7 \%)$ are highly cited papers.

Analytical chemistry plays a vital role in infectious disease diagnosis and prevention. Gram stain and culture of microorganisms are commonly used methods in microbiology. However, these conventional methods often either lack high

Published in the topical collection Analytical Chemistry for Infectious Disease Detection and Prevention with guest editors Chaoyong Yang and XiuJun (James) Li.

XiuJun Li

xli4@utep.edu

1 Department of Chemistry and Biochemistry, University of Texas at El Paso, 500 West University Ave, El Paso, TX 79968, USA

2 Border Biomedical Research Center, Environmental Science and Engineering, University of Texas at El Paso, 500 West University Ave, El Paso, TX 79968, USA

3 Key Laboratory for Chemical Biology of Fujian Province, MOE Key Laboratory of Spectrochemical Analysis \& Instrumentation, Collaborative Innovation Center of Chemistry for Energy Materials, State Key Laboratory of Physical Chemistry of Solid Surfaces, College of Chemistry and Chemical Engineering, Xiamen University, Xiamen 361005, China detection sensitivity and specificity or require a long time. Hence, molecular diagnostic methods such as genetic analysis-based quantitative polymerase chain reaction (qPCR) and immunoassay-based enzyme-linked immunosorbent assay (ELISA) have become popular in recent years. For instance, high-throughput qPCR becomes the gold standard in current COVID-19 diagnostics; and immunoassay-based antibody tests (e.g., $\operatorname{IgG}$ and $\operatorname{IgM}$ ) are also routinely used to check immunization results from COVID-19 vaccines. Along with those well-established methods, new innovative strategies including microfluidic devices, nanotechnology, and molecular imprinting have been applied to analytical chemistry to improve various aspects of biosensing such as signal amplification for high detection sensitivity, and to address new challenges for rapid point-of-care (POC) infectious disease diagnostics in low-resource settings. Therefore, we organize this paper collection and invite worldwide experts in this field to highlight recent innovations in analytical chemistry for infectious disease detection and prevention.

This collection of articles includes reviews and original research articles. Three reviews summarize the most recent advances in particular fields of analytical chemistry for infectious disease diagnostics. Recently, a lot of research efforts focus on the development of rapid and cost-effective detection approaches. In a review article, Yang and co-workers summarized important advances in the selection and detection of bacterial- and virus-associated functional nucleic acids. To address some limitations of conventional methods, in a different review article, Lu et al. reviewed recent advances in molecular imprinting (MIP) for bacterial detection, introduced the underlying recognition mechanisms, and highlighted the applications of MIP-based biosensors in sensing foodborne pathogenic bacteria. In addition, another review article from Marco et al. summarized the most recent findings of the biological implications and the clinical significance of the main Pseudomonas aeruginosa alkylquinolones (AQs) that have potential to be used as biomarkers of infections.

Two original research papers from Yingfu Li and Zhaohui $\mathrm{Li}$ et al. reported two novel methods for rapid and sensitive 
detection of SARS-CoV-2, which are based on nanotechnology and single molecule assays, respectively. $\mathrm{Wu}$ and coworkers developed an ultrasensitive colorimetric sensor array (CSA) based on the interactions between aminoglycoside antibiotics and $\mathrm{Ag}$ nanoparticles decorated with $\beta$-cyclodextrin to discriminate microorganisms quickly and accurately. Signal amplification has been pursued to improve detection sensitivity. For instance, Lin's group presented a homogeneous electrochemical biosensor for microRNA detection based on an enzyme-driven cascaded signal amplification strategy. Cabrera's group reported a novel PCR-assisted impedimetric biosensor for colibactin-encoding polyketide synthase (pks) genomic island detection in Escherichia coli samples.

POC detection of infectious diseases is a hot research field. Interestingly, Yu's group used a WiFi scanner for quantitative detection of disease markers in blood plasma, while Tokeshi's group reported a non-competitive immunoassay for detection of $\mathrm{H} 5$ avian influenza virus using a portable analyzer. Chen's group reported a lateral flow gene assay using gold nanoparticles for POC detection of African swine fever virus. Furthermore, Xiujun Li's group developed a multicolorimetric ELISA biosensor on a paper/polymer hybrid microfluidic device for visual quantitative $\mathrm{POC}$ detection of hepatitis $\mathrm{C}$ without the aid of specialized instruments such as a spectrophotometer, which has great potential for many applications particularly in resource-limited settings such as rural areas and developing nations.

We would like to thank all authors for contributing their valuable work to this topical collection. We are also grateful to the Editorial Office and Editors for their timely assistance and support. Lastly, we hope this topical collection will serve as a valuable source of information on state of the art in infectious disease detection for Anal Bioanal Chem readers.

Publisher's note Springer Nature remains neutral with regard to jurisdictional claims in published maps and institutional affiliations.

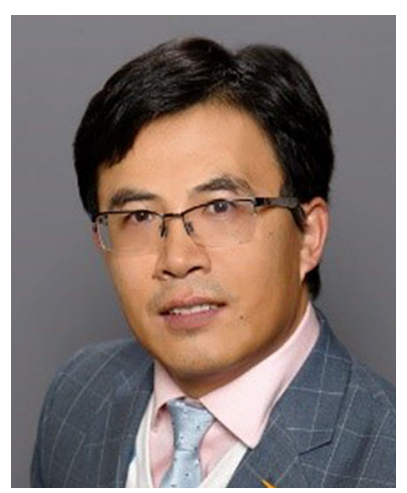

XiuJun (James) Li is Associate Professor in the Department of Chemistry \& Biochemistry at the University of Texas at El Paso (UTEP), USA. His current research interest is centered on the development of innovative microfluidic lab-on-a-chip and nanotechnology for bioanalysis, biomaterial, biomedical engineering, and environmental applications, including but not limited to low-cost diagnosis, nano-sensing, 3D cell culture, and single-cell analysis. He has coauthored about 100 publications and 22 patents, including three books from Elsevier. He is an editor of 6 journals including Scientific Reports, and the recipient of the "Bioanalysis New Investigator Award" in 2014, and also serves on the Advisory Board of Lab on a Chip and Analyst.

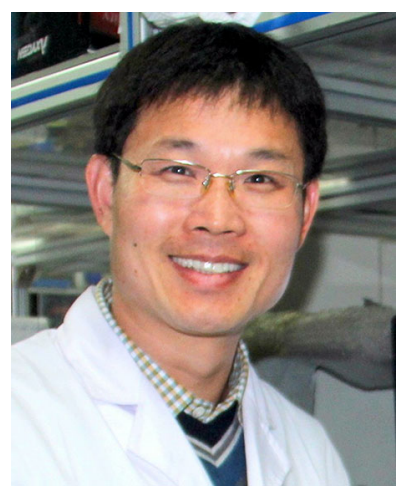

Chaoyong Yang is Distinguished Professor in the Department of Chemical Biology, Xiamen University. He received his $\mathrm{PhD}$ from the University of Florida under the guidance of Professor Weihong Tan. After postdoc training in Professor Richard Mathies' group at UC Berkeley, he joined Xiamen University in 2008. He has published more than 230 research papers in international journals which have received over 12,000 citations. He coauthored 2 books and holds 45 patents. He is Associate Editor of ACS Applied Bio Materials and has served on the advisory board of 10 international journals. He is Fellow of the Royal Society of Chemistry and a senior member of the Chinese Chemical Society. He received many awards including CAPA Distinguished Faculty Award in 2012, National Outstanding Young Investigator Award in 2013, Chinese Young Analyst Award in 2015, Chinese Chemical Society-Royal Society of Chemistry Young Chemist Award in 2016, and 2021 ACS Advances in Measurement Science Lectureship Award. His research focuses on the development of novel chemical and microfluidic tools for life science research and disease diagnosis, which integrates molecular engineering approaches, bio-nanotechnologies, and microfluidics to develop novel methods, smart materials, and functional miniaturized devices to address previously intractable bioanalytical and biomedical problems. 